\title{
Mental time in amnesia: Evidence from bilateral medial temporal damage before and after recovery
}

\author{
Shahar Arzy \\ Department of Neurology, Hadassah Hebrew University Hospital, Jerusalem, Israel, and Laboratory of Cognitive Neuroscience, Brain Mind \\ Institute, Ecole Polytechnique Fédérale de Lausanne, Lausanne, Switzerland
}

Atira Bick

Department of Neurology, Hadassah Hebrew University Hospital, Jerusalem, Israel

Olaf Blanke

The human mind is continuously involved in "projecting" the self in time in order to process past memories and predict future occurrences. "Self-projection" in time involves episodic and spatial memory, relying on medial-temporal structures, but also engages visuo-spatial imagery, relying on occipito-temporal mechanisms, and self-location, relying on temporo-parietal activity. Here we had the rare opportunity to investigate the relation between self-projection in time and memory, using a novel behavioural paradigm, in a patient with subacute bilateral medial-temporal damage during a period of amnesia as well as after recovery. Despite her memory deficit the patient was able to "project" herself to past and future, yet with significant improvement after recovery. These findings specify the relations between episodic memory and medial-temporal structures with self-projection in time to past and future.

Keywords: Mental time; Episodic memory; Medial temporal lobe; Amnesia; Limbic encephalitis.

A major aspect of the human mind is the ability to "project" the self in the mental time (MT) in order to remember the past and to predict the future (Atance \& O’Neill, 2001; Levine, 2004; Tulving, 2002). Self-projection in time is defined as "a shift of perception from the immediate environment to the alternative, imagined [past or] future

Q1 environment" (Buckner \& Carroll, 2007, p. 49), allowing to experience the self as continuous through MT: to reexperience one's personal past through the subjective "projection" of the self to a specific place and time of a past event, or to imagine a forward self-location and to preexperience a future event (Atance \& O'Neill, 2001; Tulving, 2002). MT has classically been related to memory functions, supposing that memory is needed not only to retrieve past episodic memories but also to predict future events (Addis, Wong, \& Schacter, 2007; Atance \& O’Neill, 2001; Suddendorf \& Corballis, 2007). Accordingly, Jerusalem, Israel (E-mail: shahar.arzy@hcuge.ch) 
amnesic patients with medial-temporal lesion have been found to have deficits not only in retrieval of past events (Corkin, 2002; Squire, Stark, \& Clark, 2004; Zola-Morgan, Squire, \& Amaral, 1986), but also in future prediction (Atance \& O'Neill, 2001; Hassabis, Kumaran, Vann, \& Maguire, 2007a; Rosenbaum et al., 2005; Tulving, 2002).

In a recent study (Arzy, Molnar-Szakacs, \& Blanke, 2008), we showed that besides episodic memory, processing of MT also requires the ability to "project" oneself in time in order to experience past and future events. In this study we tested self-projection in time from three different self-locations in participants' life: past, present, and future (Figure 1A). Using behavioural measures and electrical neuroimaging we showed that behaviour and brain activity are similar for self-projection to past and future self-locations. Electrical neuroimaging showed self-projection in time to activate a network of brain areas consisting of the antero-medial temporal cortex, traditionally related to memory functions (Corkin, 2002; Squire et al., 2004; Zola-Morgan et al., 1986), together with occipito-temporal cortex and temporo-parietal cortex, which are related to visual memory, self-location, and mental ownbody imagery (Addis et al., 2007; Arzy, Thut, Mohr, Michel, \& Blanke, 2006). Based on these findings of a distributed cortical network encoding for self-projection in time we hypothesized here that patients with amnesia due to medial temporal damage should still be able to project themselves in time, despite memory impairments. This would be compatible with partial dissociation between Q2 memory and self-projection in time.

\section{Patient}

The patient was a right-handed 54-year-old woman, with no history of neurological or psychiatric disorders, who suffered from voltage gated potassium channels (VGKC) antibodies associated limbic encephalitis. Limbic encephalitis is an inflammatory process that is primarily localized in the limbic system including the medial temporal lobe bilaterally. Limbic encephalitis has typically been regarded as a paraneoplastic (malignancy-related) disorder. However, in recent years a different form of limbic encephalitis has been identified that is associated with VGKC-antibodies. This type may respond to immunotherapy as administered in the present patient (Thieben et al., 2004; Vincent et al., 2004). The patient's disease manifested as a short- and long-term memory loss accompanied by personality changes with no disorientation in space or time (Table 1). The neurological examination did not show any sensorimotor deficits. The neuropsychological examination (Table 1) showed disturbance in short-term memory, working memory, episodic memory, and semantic memory, but no evidence of confabulation. Her neuropsychiatric evaluation showed moderate deficits in executive functions, including marked lability, tangentiality and disinhibition. No deficits were detected for language, attention, calculation, topography, or praxis (Table 1).

Cerebro-spinal fluid (CSF) showed high protein level $\left(92 \mathrm{mg} / \mathrm{mm}^{3}\right)$ and positive oligoclonal bands compatible with an inflammatory process. Magnetic resonance imaging (MRI) of the brain showed enhancement at the anterior and middletemporal lobe bilaterally (Figure 2A). Whole body computed tomography (CT) scan, positron emission tomography with fluorodeoxyglucose (PET-FDG), and mammography did not show any neoplasm. Immunological and paraneoplastic biomarkers in blood and CSF were negative, but antibodies for VGKC were found to be positive. The patient was treated with high-dose corticosteroids and plasma exchange. Under treatment her condition was gradually improved. Three months after treatment initiation she was able to manage again her own business, with memory functions progressively recovering (Table 1). There were no signs of lability, tangentiality, and disinhibition. MRI demonstrated almost a complete disappearance of the abovementioned signal enhancement (Figure 1B).

We tested self-projection in time during hospitalization and three months after initiation of immunomodulatory treatment. Written informed consent was obtained prior to inclusion in the study from the patient and her family. The patient and 7 age- and gender-matched control participants (mean $\pm S D, 53 \pm 4$ years) with 
(a)

first child

45 's birthday

wearing glasses

golden wedding

Challenger explosion

Hurricane Katrina

AIDS vaccination

nuclear car

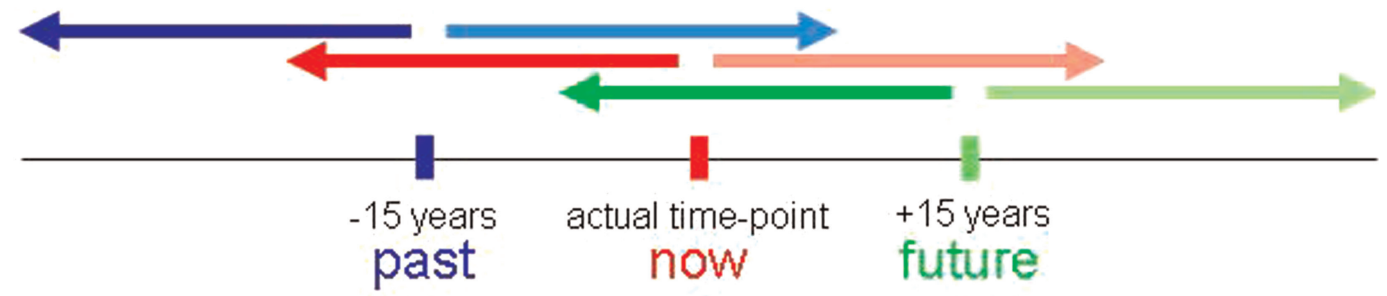

(b)
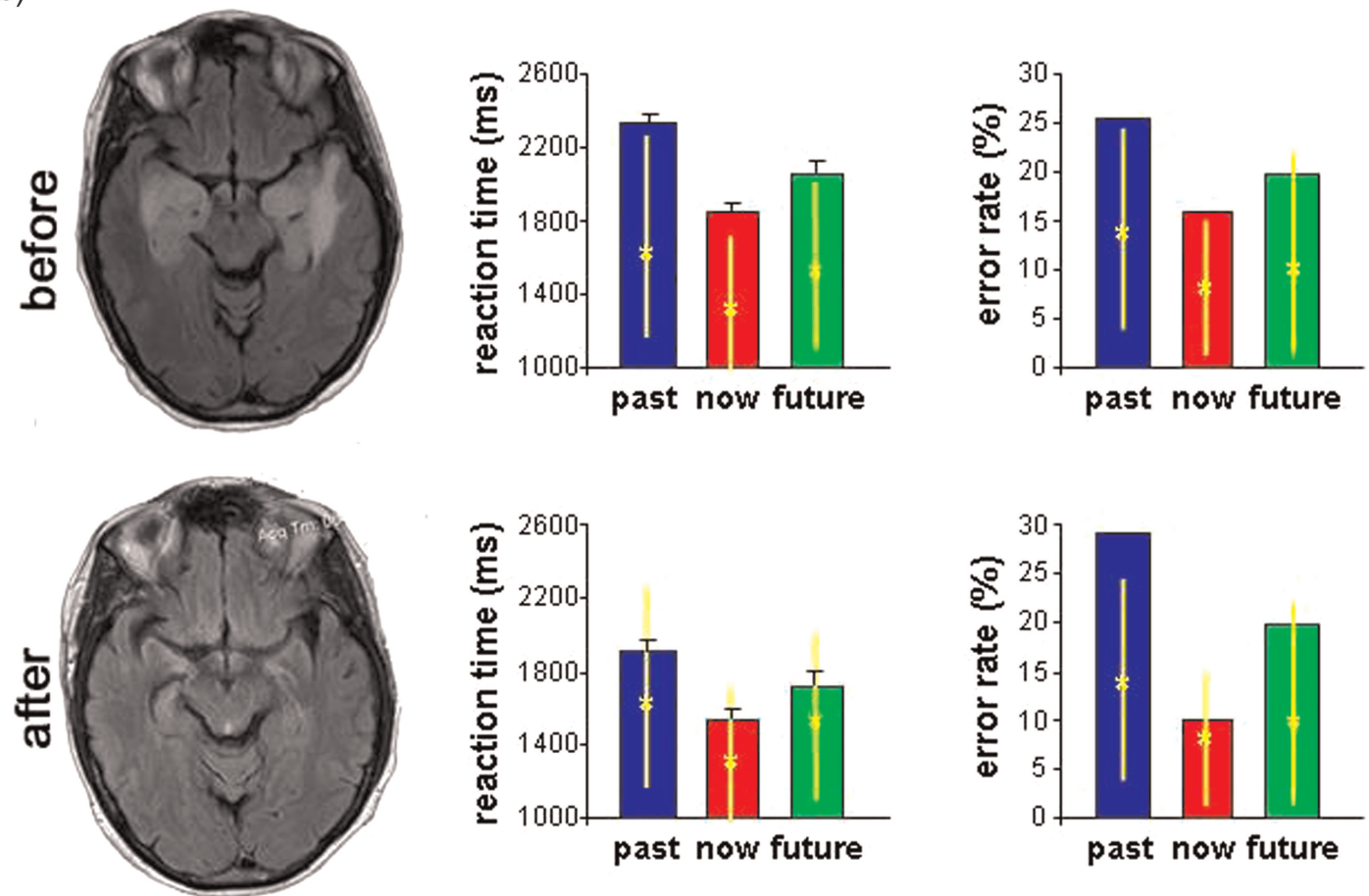

Figure 1. (A) Stimuli and procedure of the MT-task (MT= mental time). The three different self-locations in time-past, now, and future-are shown. Participants were asked to mentally "project" themselves to one of these self-locations and to judge whether different self- or non-self-related events (see, e.g., in upper row) had already happened (relative past) or were yet to bappen (relative future). (B) Patient medial-temporal damage and performance before and after recovery. Sagittal T2-FLAIR (fluid-attenuated inversion recovery) section MRI (magnetic resonance imaging) of the patient before treatment (upper row) showing bilateral inflammatory lesion at the medial temporal region, almost completely disappearing three months after treatment initiation (lower row). The bar diagrams show reaction times (left) and error rates (right) of the patient in the MT-task. Note the shorter reaction times for the now self-location than for past and future. Asterisks and lines on the bars show results (mean $\pm \mathrm{SD}$ ) for an age- and gender-matched control group. To view a colour version of this figure, please see the online issue of the Journal.

similar socio-economic and educational background as the patient were presented with different events that were taken from self-related personal history (e.g., first child, silver anniversary) or from non-self-related world occurrences (e.g., Challenger explosion, hurricane Katrina; Figure 1A). Events were taken from the list of general events used previously (Arzy et al., 2008) 
Table 1. Neuropsychological findings before treatment initiation and three months after

\begin{tabular}{|c|c|c|c|c|}
\hline \multirow[b]{2}{*}{ Test } & \multicolumn{2}{|c|}{ Before } & \multicolumn{2}{|c|}{ After } \\
\hline & Score & Impairment & Score & Impairment \\
\hline \multicolumn{5}{|l|}{ Rey Auditory-Verbal Learning Test } \\
\hline Learning & $33 / 75$ & Severe & $54 / 75$ & Percentile 40 \\
\hline Discriminability & $11 / 15$ & Severe-moderate & $15 / 15$ & Percentile 90 \\
\hline Delayed recall & $5 / 15$ & Severe & $9 / 15$ & Percentile 10 \\
\hline \multicolumn{5}{|l|}{ Rey Visual Learning Test } \\
\hline Trial 1 & $7 / 15$ & Severe-moderate & $12 / 15$ & Mild \\
\hline Trial 2 & $6 / 15$ & Severe-moderate & $12 / 15$ & Mild \\
\hline \multicolumn{5}{|l|}{ Addenbrooke's Cognitive Examination } \\
\hline Orientation & $10 / 10$ & - & $10 / 10$ & - \\
\hline Attention \& Concentration & $8 / 8$ & - & $8 / 8$ & - \\
\hline Memory 1 & $3 / 3$ & - & $3 / 3$ & - \\
\hline \multirow{5}{*}{$\begin{array}{cc}\text { Memory } 2 & \text { Trial } 1 \\
& \text { Trial } 2 \\
& \text { Trial } 3 \\
\text { 5-Min Delayed Recall } \\
\text { 20-Min Delayed Recall }\end{array}$} & $4 / 7$ & Moderate & $5 / 7$ & Mild \\
\hline & $5 / 7$ & Moderate & $7 / 7$ & - \\
\hline & $6 / 7$ & Mild & $7 / 7$ & - \\
\hline & $7 / 7$ & - & $7 / 7$ & - \\
\hline & $6 / 7$ & Mild & $7 / 7$ & - \\
\hline Retrograde Memory & $1 / 4$ & Severe & $4 / 4$ & - \\
\hline Verbal Fluency-Animals (scaled score) & $5 / 7$ & Moderate & $6 / 7$ & Mild \\
\hline Verbal Fluency-Letters (scaled score) & $6 / 7$ & Mild & $6 / 7$ & Mild \\
\hline Naming & $11 / 12$ & Mild & $12 / 12$ & - \\
\hline Language Comprehension & $8 / 8$ & - & $8 / 8$ & - \\
\hline Repetition & $5 / 5$ & - & $5 / 5$ & - \\
\hline Reading & $2 / 2$ & - & $2 / 2$ & - \\
\hline Writing & $1 / 1$ & - & $1 / 1$ & - \\
\hline Visual Spatial/Praxis & $5 / 5$ & - & $5 / 5$ & - \\
\hline Total score & $86 / 100$ & Mild-moderate & $96 / 100$ & \\
\hline Frontal Assessment Battery & $15 / 18$ & Mild & $18 / 18$ & - \\
\hline Cookie Theft & $2 / 2$ & - & $2 / 2$ & - \\
\hline Map Reading & $7 / 7$ & - & $7 / 7$ & - \\
\hline Benton Facial Recognition & $37 / 50$ & Moderate & $45 / 50$ & \\
\hline \multicolumn{5}{|l|}{ Attention and Concentration } \\
\hline $20-1$ & 0 error & - & 0 error & - \\
\hline $\mathrm{ABC}$ & 0 error & - & 0 error & - \\
\hline Serial threes & 0 error & - & 0 error & - \\
\hline
\end{tabular}

and were adapted to the patient according to her past history and plans as were obtained at admission and during neuropsychological testing, as well as by complementary information from family members. Stimuli appeared for $1,000 \mathrm{~ms}$ in the centre of the computer screen with an interstimulus interval of 2,000 ms. Judgements were given using index and middle fingers of the left and right hand in alternating blocks as a button press on a serial response box. Patient and control participants were instructed to respond as quickly and precisely as possible while maintaining a mental image of themselves in the appropriate temporal self-location (past, now, or future); this was performed in three blocks. Each block included 60 stimuli, equally distributed among four groups: self-related (personal events) in relative past, self-related in relative future, non-selfrelated (world events) in relative past, and nonself-related in relative future, appearing in random order. Stimuli were presented, and reaction time and accuracy were recorded, using 
E-Prime ${ }^{\circledR}$ software (Schneider, Eschman, \& Zuccolotto, 2002). Patient and, accordingly, controls were asked first to judge from their present self-location in time (now) whether these events had already happened (relative past) or were yet to happen (relative future). They were then asked to imagine themselves 15 years younger (past self-location) or older (future self-location) and to make the same judgements (Figure 1A). In each block (past, now, and future), patient and controls were repeatedly instructed in the beginning of each session to imagine themselves in a specific past, present, or future time and from this time point to make "relative-past" or "relative-future" judgements about the presented events. While the explicit measurement relates to relative past versus relative future, the task implicitly measures self-projection in time, which is the main interest in the present patient. Subsequent to the experiment patient and controls were asked to note for each event how long ago this event took place (for past events) or in how many years it was supposed to happen. These responses were used to quantify the degree of memory impairment (percentage of mistaken or missing responses), as well as to determine correct responses for each participant. Patient responses were verified according to complementary anamnesis by family members. While control participants could recall 100\% of both self- and non-self-related events, the patient, before treatment initiation, could explicitly recall $78 \%$ of the self-related events and $31 \%$ of the non-self-related events. Three months after treatment initiation the patient showed important recovery and recalled all self-related events and $68 \%$ of the non-self-related events (for further neuropsychological details see Table 1).

The patient was tested with the abovedescribed MT-task before treatment initiation and three months afterwards. Testing before treatment initiation showed that reaction times in past and future self-locations were significantly higher than those in the now (MT effect: past, $2,334 \pm 60 \mathrm{~ms} ; \quad$ now, $1,841 \pm 47 \mathrm{~ms} ;$ future, $2,056 \pm 63 \mathrm{~ms})$. A similar pattern was found in an age- and gender-matched control group
(Figure 1B, upper row). Behavioural results also showed an effect of self (self-related faster than non-self-related events: self-related, 1,890 \pm $59 \mathrm{~ms}$; non-self-related, $2,227 \pm 63 \mathrm{~ms}$ ), as was found also in the control group. Error rates also showed both effects (MT effect: past, 29\%; now, 9\%; future, 21\%; self effect: self-related, 14\%; non-self-related, 24\%). Testing three months after treatment showed effects of MT (past, $1,947 \pm 60 \mathrm{~ms}$; now, $1,589 \pm 42 \mathrm{~ms}$; future, $1,739 \pm 66 \mathrm{~ms}$ ) and self (self-related, 1,643 \pm $56 \mathrm{~ms}$; non-self-related, $1,939 \pm 59 \mathrm{~ms}$ ), comparable to repeated testing in the control group (Figure 1B, lower row). Error rates also showed both effects (MT: past, 26\%; now, 19\%; future, 20\%; self: self-related, $19 \%$; non-self-related, 24\%). Statistical analysis of reaction times showed main effects of treatment, before and after, $F(1,3)=82.4, p<.001$, MT, $F(2,6)=$ 230.7, $p<.001$, and self, $F(1,3)=37.9, p<$ .01 , with an interaction only for Recovery $\times$ MT, $F(2,6)=19.9, p<.005$ (see appendices for more statistical and post hoc tests). Statistical analysis of reaction times for the control group showed effects of MT, $F(2,12)=12.7, p<.01$, and self, $F(1,6)=4.6, p<.05$, with no effect of repeated testing, $F(1,6)=0.5, p=.48$. Similar results were found for error rates: MT, $F(2,12)$ $=4.0, p<.05$; self, $F(1,6)=29.1, p<.01$; repeated testing, $F(1,6)=0.4, p=.8$. Finally, in order to exclude a mental calculation strategy to account for our results we checked 5 additional participants in the MT-task, yet asking them to use mental calculation strategy and not self-projection in time. None of these participants was able to perform the task in the given interstimulus interval of 2,000 ms. In addition, patient (after recovery) and controls showed a tendency to be faster for future events than for past events. Taken together, these results show that mental calculation cannot account for the behavioural results recorded in the MT-task.

\section{Discussion}

Patients with amnesia suffer from a disturbance in acquiring new memories (anterograde amnesia) or

COGNITIVE NEUROPSYCHOLOGY, 0000, 00 (0) 
recall past memories (retrograde amnesia), generally attributed to medial temporal lesions (Corkin, 2002; Squire et al., 2004; Zola-Morgan et al., 1986), compatible with the current patient's symptoms and brain damage. Here we investigated the relation between memory and self-projection in time in an amnesic patient. We report the following novel observations: (a) preservation of self-projection in time in amnesia; (b) similar behavioural results for self-projection in time to past and future in amnesia; (c) difference in performance of self-projection in time with and without amnesia. These observations are discussed with respect to the role of medial temporal structures and other brain regions in episodic memory and self-projection in time.

A central component in processing of MT is the ability to project the self forward in time to preexperience an event and to project the self backward to reexperience it. This is supposed to be related to episodic memory, as the system that allows us to remember personally experienced events and to reexperience those events (Atance \& O’Neill, 2001; Schacter, Addis, \& Buckner, 2007). It also requires self-location mechanisms orienting the self on a time-line and with respect to these events (Arzy et al., 2008; Bird \& Burgess, 2008; Burgess, Becker, King, \& O'Keefe, 2001; Moscovitch et al., 2005; Spreng, Mar, \& Kim, 2008).

The medial temporal region is the most crucial structure in acquiring both semantic and episodic memory, and damage to this region might cause amnesia (Corkin, 2002; Squire et al., 2004; ZolaMorgan et al., 1986). However, self-projection in time has been shown previously to rely not only on memory-related structures in the anteromedial temporal lobe, but also on structures in occipito-temporal cortex related to visual memory (Addis et al., 2007; Arzy et al., 2008) and structures in temporo-parietal cortex related to mental own-body imagery and self-location (Arzy et al., 2008; Levine, 2004). We hypothesize that these brain regions outside the medial temporal lobe that were not affected in the patient might also contribute to her ability to project herself in time despite of her antero-medial cortex deficit. This hypothesis is also supported by the dissociation between the ability to recall events (declarative memory) and to refer to these memories (nondeclarative memory); in medial temporal damage, the former is mostly impaired, showing the role of the medial temporal lobe in declarative rather then nondeclarative memory (Corkin, 2002; Squire et al., 2004; Zola-Morgan et al., 1986). Self-projection in time might be related to nondeclarative memory, relying on extratemporal mechanisms, and this might explain the preservation of our patient's ability to solve the MT-task, although she was only partially able to explicitly recall the same events. This is also supported by the fact that memory for non-selfrelated world occurrences, which probably relies on a functions other than episodic and semantic memories, was much worse than memory for self-related events prior to treatment.

There was a significant difference in self-projection in time before and after the patient's recovery, as was also shown by a significant interaction between these two factors. Although we cannot exclude that this improvement is related to unspecific mechanisms (such as difficulty, fatigue, lack of concentration, or "test-retest" effect), we do not think that this is very likely because the patient was well motivated and responded during both test phases, and none of our control participants showed comparable test-retest effects. In addition, three months elapsed between both tests for patient and controls. Moreover, other memory tasks also showed improvement after recovery, but this was not observed for attention, language, or visuospatial tasks (Table 1). We would rather suggest that self projection in time relies on distributed neural network, in which the anteromedial temporal cortex also plays a role. This function might be related to its above-mentioned role in episodic and semantic memory (Corkin, 2002; Squire et al., 2004; Zola-Morgan et al., 1986), but also to its role in imagining experiences through the provision of spatial context of the events (Bird \& Burgess, 2008; Burgess et al., 2001; Burgess, Maguire, \& O’Keefe, 2002; Hassabis, Kumaran, Vann, \& Maguire, 2007b; Moscovitch et al., 2005; Spreng et al., 2008), or 
"scene construction", involving the generation, maintenance, and visualization of complex spatial contexts (Hassabis \& Maguire, 2007), which has also been shown to be critically reliant on the hippocampus and wider medial temporal lobe (Hassabis et al., 2007a, 2007b).

In conclusion, this study supplies causal evidence that bilateral medial-temporal damage, although impairing memory functions, does not demolish the ability to mentally project the self to different self-locations in time, in past, present, or future. This ability was similar with respect to past and future before and after recovery. However, this "self-projection" was much faster after recovery, showing a partial contribution of medial temporal lobe mechanisms to self-projection in time. These findings contribute to understanding self-projection in time and its neural bases, and to segregating the brain mechanisms of episodic memory and temporal self-location in health and disease.

Manuscript received 14 November 2008 Revised manuscript received 8 October 2009 Revised manuscript accepted 19 October 2009 First published online day month year

\section{REFERENCES}

Addis, D. R., Wong, A. T., \& Schacter, D. L. (2007). Remembering the past and imagining the future: Common and distinct neural substrates during event construction and elaboration. Neuropsychologia, 45(7), 1363-1377.

Arzy, S., Molnar-Szakacs, I., \& Blanke, O. (2008). Self in time: Imagined self-location influences neural activity related to mental time travel. Journal of Neuroscience, 28(25), 6502-6507.

Arzy, S., Thut, G., Mohr, C., Michel, C. M., \& Blanke, O. (2006). Neural basis of embodiment: Distinct contributions of temporoparietal junction and extrastriate body area. Journal of Neuroscience, 26(31), 8074-8081.

Atance, C. M., \& O'Neill, D. K. (2001). Episodic future thinking. Trends in Cognitive Science, 5(12), 533-539.

Q4 Bird, C. M., \& Burgess, N. (2008). The hippocampus and memory: Insights from spatial processing. Nature Reviews Neuroscience.
Buckner \& Carroll, 2007

Burgess, N., Becker, S., King, J. A., \& O'Keefe, J. (2001). Memory for events and their spatial context: Models and experiments. Philosophical Transactions of the Royal Society of London. Series B, Biological Sciences, 356(1413), 1493-1503.

Burgess, N., Maguire, E. A., \& O'Keefe, J. (2002). The human hippocampus and spatial and episodic memory. Neuron, 35(4), 625-641.

Corkin, S. (2002). What's new with the amnesic patient H.M.? Nature Reviews Neuroscience, 3(2), 153-160.

Hassabis, D., Kumaran, D., \& Maguire, E. A. (2007a). Using imagination to understand the neural basis of episodic memory. Journal of Neuroscience, 27(52), 14365-14374.

Hassabis, D., Kumaran, D., Vann, S. D., \& Maguire, E. A. (2007b). Patients with hippocampal amnesia cannot imagine new experiences. Proceedings of the National Academy of Sciences USA, 104(5), 17261731.

Hassabis, D., \& Maguire, E. A. (2007). Deconstructing episodic memory with construction. Trends in Cognitive Science, 11(7), 299-306.

Levine, B. (2004). Autobiographical memory and the self in time: Brain lesion effects, functional neuroanatomy, and lifespan development. Brain and Cognition, 55(1), 54-68.

Moscovitch, M., Rosenbaum, R. S., Gilboa, A., Addis, D. R., Westmacott, R., Grady, C., et al. (2005). Functional neuroanatomy of remote episodic, semantic and spatial memory: A unified account based on multiple trace theory. Journal of Anatomy, 207(1), 35-66.

Rosenbaum, R. S., Kohler, S., Schacter, D. L., Moscovitch, M., Westmacott, R., Black, S. E., et al. (2005). The case of K.C.: Contributions of a memory-impaired person to memory theory. Neuropsychologia, 43(7), 989-1021.

Schacter, D. L., Addis, D. R., \& Buckner, R. L. (2007). Remembering the past to imagine the future: The prospective brain. Nature Reviews Neuroscience, 8(9), 657-661.

Schneider, W., Eschman, A., \& Zuccolotto, A. (2002). E-Prime user's guide. Pittsburgh, PA: Psychology Software Tools.

Spreng, R. N., Mar, R. A., \& Kim, A. S. (2008). The common neural basis of autobiographical memory, prospection, navigation, theory of mind and the default mode: A quantitative meta-analysis. Journal of Cognitive Neuroscience.

COGNITIVE NEUROPSYCHOLOGY, 0000, 00 (0)
Q1 
Squire, L. R., Stark, C. E., \& Clark, R. E. (2004). The medial temporal lobe. Annual Reviews of Neuroscience, 27, 279-306.

Suddendorf, T., \& Corballis, M. C. (2007). The evolution of foresight: What is mental time travel, and is it unique to humans? Behavioral and Brain Sciences, 30(3), 299-313; discussion 313-251.

Thieben, M. J., Lennon, V. A., Boeve, B. F., Aksamit, A. J., Keegan, M., \& Vernino, S. (2004). Potentially reversible autoimmune limbic encephalitis with neuronal potassium channel antibody. Neurology, 62(7), 1177-1182.

\section{Interactions and post hoc tests (Newman- Keuls) for patient's performance}

\begin{tabular}{lllcc}
\hline Effect & \multicolumn{1}{c}{ Variables } & $F$ & $p$ \\
\hline Interaction & & Treatment $\times$ Self & 0.75 & .45 \\
& & MT $\times$ Self & 0.80 & .49 \\
& & Treatment $\times$ MT $\times$ Self & 5.6 & $<.05^{*}$ \\
\multirow{2}{*}{ Treatment } & Past & Before vs. after & & $<.05^{*}$ \\
& Now & Before vs. after & & $<.05^{*}$ \\
& Future & Before vs. after & $<.05^{*}$ \\
MT & Before & Past vs. now & $<.05^{*}$ \\
& & Now vs. future & $<.05^{*}$ \\
& & Past vs. future & .4 \\
& After & Past vs. now & $<.05^{*}$ \\
& & Now vs. future & $<.05^{*}$ \\
& & Past vs. future & .7 \\
\hline
\end{tabular}

Note: $\mathrm{MT}=$ mental time.
Tulving, E. (2002). Episodic memory: From mind to brain. Annual Reviews of Psychology, 53, 1-25.

Vincent, A., Buckley, C., Schott, J. M., Baker, I., Dewar, B. K., Detert, N., et al. (2004). Potassium channel antibody-associated encephalopathy: A potentially immunotherapy-responsive form of limbic encephalitis. Brain, 127(3), 701-712.

Zola-Morgan, S., Squire, L. R., \& Amaral, D. G. (1986). Human amnesia and the medial temporal region: Enduring memory impairment following a bilateral lesion limited to field CA1 of the hippocampus. Journal of Neuroscience, 6(10), 2950-2967.

\section{APPENDIX B}

Interactions and post hoc tests for controls' performance

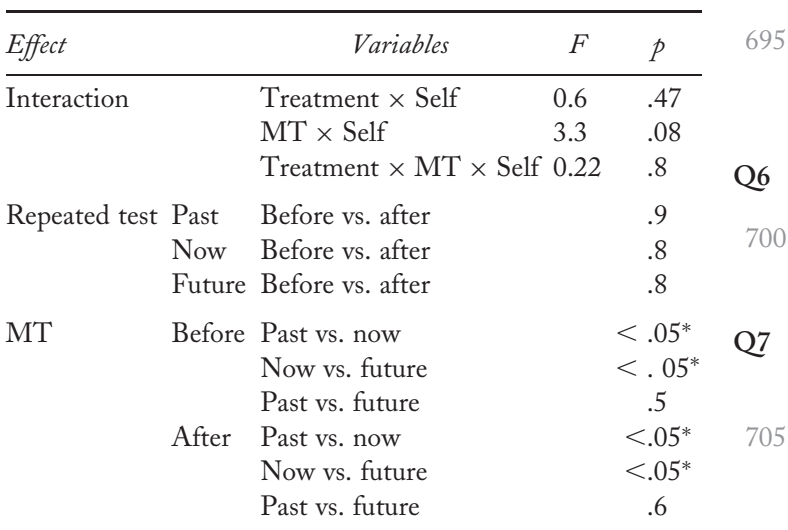

Note: $\mathrm{MT}=$ mental time. 